\title{
Aplicación de la mecánica de fluidos en la modelación matemática de flujo de SO2 en el Centro Histórico de Riobamba - Ecuador.
}

Application of fluid mechanics in the mathematical modeling of SO2 flow in the Historical Center of Riobamba - Ecuador

Javier Roberto Mendoza Castillo. ${ }^{1}$, Jenner José Baquero Luna. ${ }^{2}$, Esteban Ricardo Baquero Aldaz. $^{3}$, Romel Manolo Insuasti Castelo. ${ }^{4}$

Recibido: 10-02-2019 / Revisado: 25-02-2019 /Aceptado: 14-03-2019/ Publicado: 05-04-2019

\begin{abstract}
.
DOI: https://doi.org/10.33262/cienciadigital.v3i2.467

Atmosphere in high altitude cities like Riobamba (2754 masl) behaves in a difficult way to predict because the pressure, temperature, wind direction and thermal inversions are erratic. An interdisciplinary team consisting of mathematicians, a physicist, a statistician and a chemist, during 4 years carried out studies to determine if the SO2 concentration was related to respiratory diseases in the city; This article is part of the aforementioned studies, for which SO2 monitoring was carried out with equipment from accredited laboratories in Ecuador; the data were submitted to statistical analysis, establishing mathematical models of smoothing and finally the differential equations that characterize the flow of SO2 from one of the chaotic sites of the city by the accumulation of mobile sources were deduced.
\end{abstract}

Keywords: Fluid Mechanics, Differential Equations, Mathematical Models, Air Pollution, Environment.

\section{Resumen.}

\footnotetext{
Escuela Superior Politécnica de Chimborazo, Facultad de Ciencias Pecuarias, Chimborazo Ecuador jmendoza@espoch.edu.ec

2 Escuela Superior Politécnica de Chimborazo, CIDED, Facultad de Ciencias, Chimborazo Ecuador, jose.baquero@espoch.edu.ec

3 Universidad Nacional de Córdoba, Córdoba Argentina, esteban.baquero@unc.edu.ar

4 Escuela Superior Politécnica de Chimborazo, Facultad de Mecánica, Chimborazo, Ecuador romel.insuasti@espoch.edu.ec
} 
La atmósfera en las ciudades de altura como Riobamba (2754 msnm) se comporta de una manera difícil de predecir debido a que la presión, temperatura, dirección del viento e inversiones térmicas son erráticas. Un equipo interdisciplinario formado por matemáticos, un físico, un estadístico y un químico, durante 4 años realizaron sendos estudios para determinar si la concentración de SO2 se relacionaba con las enfermedades respiratorias en la ciudad; el presente artículo forma parte de los mencionados estudios, para lo cual se realizaron monitoreos de $\mathrm{SO} 2$ con equipos de laboratorios acreditados en el Ecuador; se sometieron los datos a análisis estadístico, estableciendo modelos matemáticos de suavizado y finalmente se dedujeron las ecuaciones diferenciales que caractericen el flujo de SO2 desde uno de los sitios caóticos de la ciudad por la acumulación de fuentes móviles

Palabras clave: Mecánica de Fluidos, Ecuaciones Diferenciales, Modelos Matemáticos, Contaminación Del Aire, Medio Ambiente

\section{Introducción.}

El problema que motivó la presente investigación es la siguiente: ¿es posible modelar el flujo de $\mathrm{SO}_{2}$ en el Parque Maldonado de Riobamba?, punto caótico de acumulación de fuentes móviles (1) debido a su ubicación geográfica y cercanía con el casco comercial, colonial y administrativo de la ciudad.

La situación socio ambiental referida al aire en la ciudad de Riobamba (2), se basa en consecuentes efectos, riesgos y amenazas sobre la salud y el ambiente (3), debido al deterioro de la calidad del aire, causado por los problemas por una inadecuada gestión ambiental de las actividades industrial, comercial, distribución de combustibles (4), transporte, botadero de basura a cielo abierto que originan y emiten contaminantes a la atmósfera sin control; por otro lado el acelerado crecimiento poblacional, las pautas de consumo, desarrollo tecnológico y económico, urbanización no planificada y su consecuente problemática de contaminación del aire (5).

Varias actividades antropogénicas de impacto influyen en la calidad del aire de la ciudad, diferenciadas estas como fuentes fijas y móviles(6), las mismas constituyen el mayor aporte de la contaminación atmosférica en especial para el centro histórico de la ciudad de Riobamba.

Se requiere una permanente evaluación de los potenciales impactos en la salud relacionados con la contaminación del aire (7), especialmente cuando se trata de sustancias que tienen una patología tóxica y peligrosa sobre la salud humana (8), ecosistemas y ambiente, así como de gases de efecto invernadero y agotadores de la capa de ozono, siendo a la vez agentes del 
cambio climático global, los que inciden en el deterioro del ambiente atmosférico en la ciudad de Riobamba - Ecuador.

Revisando diferentes tendencias en cuanto al estudio del flujo de $\mathrm{SO} 2$ en la literatura científica en los últimos 4 años, se determina que el interés primordial en cuanto a este fenómeno es el efecto del gas mencionado gracias a las emisiones de los distintos volcanes; se registran algunos destacados ejemplos:

- Reconstruction of flux and altitude of volcanic SO2 emissions from IASI satellite observations: implications for volcanological and atmospherical studies. Publicado y disertado en 2016 en: 4th IASI International Conference.(9)

- The 1994-2001 eruptive period at Rabaul, Papua New Guinea: Petrological and geochemical evidence for basalt injections into a shallow dacite magma reservoir, and significant SO2 flux, publicado en el Journal of Volcanology and Geothermal Research. (10)

- Estimación de las emisiones de dióxido de azufre-SO2, asociadas con el crecimiento de un domo de lava en el volcán Galeras en 2008. Publicado en el Boletín de Geología. (11)

- Comparación del flujo de emisión de SO2 derivadas de COSPEC y MODIS y su complementariedad en el monitoreo volcánico: caso de estudio en el Volcán Popocatépetl (México), publicado en el Boletín de la Sociedad Geológica Mexicana. (12)

Erupción del 3 de marzo de 2015 en el volcán Villarrica caracterización del lahar del estero correntoso y cuantificación de emisiones post-eruptivas de SO2 (Doctoral dissertation, Universidad de Concepción. Facultad de Ciencias Químicas. Departamento de Ciencias de la Tierra (13)

\section{EL SO2}

El dióxido de azufre (SO2) se utiliza principalmente en el blanqueo y la conservación de alimentos. El trióxido de azufre (SO3) se usa en el proceso de producción de ácido sulfúrico (14)

La principal fuente de óxidos de azufre es la quema de carbón y el combustible (dependiendo del porcentaje de azufre en el material de combustión) (15).

Fuentes adicionales de óxidos de azufre: industria del papel, procesos de refinación y fundición de metales (16)

\section{Efectos a la salud:}

La exposición a los óxidos de azufre es causada por la respiración, el contacto o la ingestión (17) . 
La exposición respiratoria al azufre puede dañar las vías respiratorias, durante 5 a 15 minutos para el dióxido de azufre a concentraciones variables de 10-50 ppm puede causar irritación en los ojos y la nariz, sensación de asfixia y tos (18).

La exposición respiratoria crónica a bajas concentraciones de dióxido de azufre puede causar inflamación respiratoria e incluso daño pulmonar. El contacto con dióxido de azufre líquido (especialmente cuando se trabaja en el lugar de trabajo) puede causar quemaduras y pérdida de la visión.

\section{Impacto ambiental:}

Los óxidos de azufre causan lluvia ácida, que afecta la flora y los sistemas de fauna en el suelo y el agua.

\section{Modelos matemáticos y ecuaciones diferenciales.}

Para hablar de modelos matemáticos debemos comprender algunos términos que son muy utilizados como modelo, sistema, sistema dinámico, entre otros.

La palabra modelo se deriva de la palabra latina modus que significa medir. En la actualidad, la palabra modelo o en plural modelos, tiene un significado muy amplio y su utilización se da en los más variados campos de la actividad humana. Evidencia la representación explícita de la forma en que una parte de la realidad es aproximada por quien analiza esa situación y que obedecen a un objetivo, una meta, una finalidad, un propósito. La palabra modelo está asociada con términos tales como patrón, prototipo, molde, ejemplar, comportamiento, trayectoria debidamente establecida o normada, entre otros (19).

La palabra sistema tiene igualmente un significado muy amplio que depende de la actividad humana. Un sistema se puede comprender como un conjunto de ideas, de métodos, de procedimientos, de elementos, de objetos o de componentes o de combinaciones de partes o de todos estos en interacción dinámica, organizada en función de un objetivo o que actúan para cumplir una tarea o que son agrupados por su finalidad, o para asegurar su funcionamiento, su éxito.

En el ámbito de la física, un sistema puede ser un conjunto de ideas científicas como por ejemplo un sistema newtoniano. El sistema internacional de unidades (SI) que consiste en siete unidades de medida métrico decimal que son: metro, kilogramo, segundo, kelvin, mole, amperio y candela. Pueden ser elementos reunidos de modo a formar un conjunto en interacción dinámica como el sistema solar, un sistema dinámico constituido por un conjunto de masas-resortes.

En el ámbito anatómico y fisiológico del cuerpo humano y de otras especies, un sistema puede estar constituido por elementos estructurados en interacción dinámica como el sistema nervioso, el sistema cardiovascular, el sistema digestivo. 
En el contexto de la estructura del país, un sistema puede ser un conjunto de métodos, de procedimientos normados para asegurar un funcionamiento colectivo como el sistema educativo, el sistema de defensa, sistema de salud. Puede ser también un conjunto de leyes, reglamentos, normas que están vigentes para establecer el modo de gobernar, de administrar, de organizar los gobiernos seccionales, provinciales y nacional, así tendremos el sistema judicial, el sistema electoral, el sistema de seguridad social, el sistema penitenciario.

En matemática, un sistema puede ser un conjunto de elementos en estructuras definidas como el sistema de los números reales, los sistemas de ecuaciones lineales o no lineales que es un conjunto de ecuaciones que relacionan simultáneamente varias variables, se habla también del sistema de referencia euleriano y lagrangeano, de los sistemas de coordenadas cartesianas y polares. Un área de estudio en amplio crecimiento y que vinculan a muchas áreas del conocimiento humano como las ciencias económicas, las ciencias naturales son los sistemas dinámicos(20).

En el campo de la informática, un sistema experto consiste en un programa computacional elaborado para resolver problemas específicos que canaliza las soluciones en función de los conocimientos acumulados.

Los sistemas pueden ser de naturaleza diversa.

- Vivientes como por ejemplo personas, colonias de hogos, bacterias, pájaros, plantas.

- Materiales como máquinas, laboratorios, equipos, medios de transporte, entre otros.

- Abstractos que pueden ser por ejemplo un modelo numérico, un programa computacional para simulación de un problema, leyes, normas, ordenanzas, entre otros.

Un sistema está estructuralmente compuesto de cuatro componentes que se describen a continuación.

1. Un límite que define las fronteras del sistema, su interior y le separa del exterior.

2. Elementos o componentes que pueden ser enlistados y estructurados por categorías, o por jerarquía o importancia dentro del sistema.

3. Fuentes o acumuladores en los que los elementos son estructurados y en los cuales son agrupados o almacenados.

4. Red de comunicación que permite los intercambios de energía, de información, de materia entre los diferentes elementos o componentes del sistema.

Interesa todo aquello que es observable, las interacciones con el exterior. Se debe precisar todo aquello que influenciará sobre el sistema, sea en forma muy significativa o que aportan en forma débil. Aquello que no es observable para los propósitos del sistema no tiene ningún interés. Es la finalidad del conjunto la que caracteriza al sistema (21).

Se tienen tres componentes importantes de un sistema: las entradas, las salidas y las perturbaciones. 


\section{Entradas}

Un sistema puede ser influenciado por un aporte de energía, de materia, de información. Las acciones exteriores que actúan sobre el sistema son llamadas entradas. Las causas que producen estas acciones son a investigar en el ambiente que rodea al sistema.

Ejemplos de entradas son: acciones, causas, datos, medios disponibles para influenciar el sistema, magnitudes que pueden ser manipuladas.

\section{Salidas}

Las acciones del sistema sobre el ambiente, las magnitudes observadas o medidas son definidas como las salidas del sistema.

Son ejemplos de salida: reacciones, efectos, resultados, comportamientos que deben ser observados o modificados, influencias del sistema sobre el ambiente, respuesta del sistema, observaciones.

\section{Perturbaciones}

Estas son como las entradas, por lo tanto, acciones que ejercen sobre el sistema pero que no son generalmente conocidas a priori. En la mayoría de los casos, las perturbaciones tienen un comportamiento imprevisible, aleatorio. Las entradas conocidas pero omitidas son consideradas como perturbaciones. Las perturbaciones son medidas que el observador no puede o no quiere manipular. En algunos casos pueden ser despreciables y en otros pueden ser muy influyentes o dominantes en el sistema.

Son perturbaciones: influencias del ambiente sobre el sistema (desconocidas, no deseadas, omitidas, aleatorias).

En el siguiente diagrama se ilustra el esquema de un sistema dinámico.

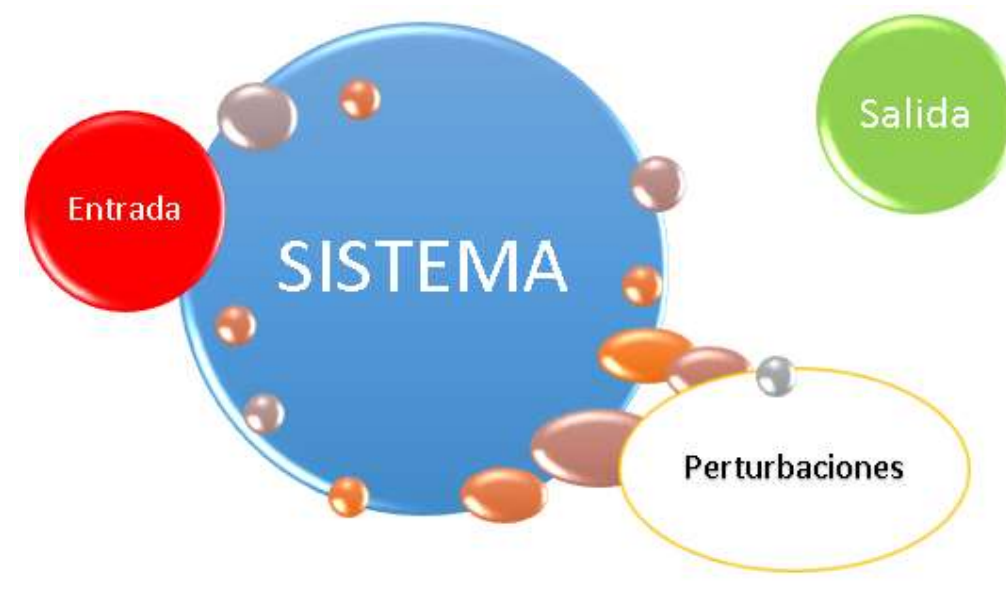

Fig. 1 Sistema dinámico 


\section{Modelos matemáticos}

Según la naturaleza del sistema se le asigna un nombre al modelo. Por ejemplo, hablaremos de modelos matemáticos, modelos de visión computacional, modelos educativos, modelos de gestión administrativa, modelos econométricos, modelos de producción agrícola, modelos físicos, modelos de desarrollo urbano, modelos de tráfico y transportes, modelos de gestión ambiental, modelos matemáticos de transporte de contaminantes en suelos y ríos, entre otros.

Más precisamente, nos interesan los modelos matemáticos y todo aquello relacionado con la simulación numérica de esta clase de problemas.

Los modelos matemáticos que consideraremos tienen como entradas y salidas elementos que son números reales. No consideramos modelos de tipo cualitativo.

De manera general, los modelos matemáticos consisten en un conjunto de relaciones matemáticas, de ecuaciones matemáticas, de relaciones lógicas que representan aproximaciones de interacciones de elementos, objetos o componentes de una estructura dinámica, de una realidad en función de un objetivo. Estos modelos describen un intercambio de información entre las variables dependientes, independientes que pueden ser de diferente naturaleza.

Los modelos matemáticos son aproximaciones de la realidad. Los modelos vienen en una diversidad de formas y sirven a una variedad de propósitos. Pueden servir como medios de predicción explícita, como herramientas de selección, pueden contribuir en la comprensión de patrones observados, son los generadores de resultados para alimentar los sistemas integrados de gestión. Pueden incorporar resultados de otras disciplinas científicas en forma coherente y orgánica.

Es un reto para el modelador, el seleccionar el mejor modelo que le permita comprender, explicar y proveer de soluciones de un determinado problema.

Por otro lado, los parámetros son cantidades que caracterizan al sistema a ser modelado. Estas cantidades pueden estar definidas en forma dimensional, esto es, tienen unidades de medida, o en su defecto, pueden ser adimensionales. Además, los parámetros pueden ser obtenidos directamente de mediciones en el propio sistema, o pueden ser obtenidas por el comportamiento real del sistema. Cada ecuación usada debe ser dimensionalmente consistente o también dimensionalmente homogénea, es decir que cada término de la ecuación tiene la misma dimensión neta. Esto se logra estableciendo la dimensión, así como sus unidades de cada una de las constantes, parámetros o variables dependientes o independientes que intervienen en el fenómeno.

Resultados de modelos matemáticos son: funciones, ecuaciones. 
Los modelos matemáticos que propone la presente investigación son un conjunto de ecuaciones que se relacionan entre sí, en dependencia con un fenómeno físico específico, que resuelve o predice un acontecimiento sujeto a comprobación, en este proceso es sumamente importante identificar qué principio físico rige y cuáles son las variables a considerar. Estas ecuaciones son utilizadas en lo posterior para resolver problemas similares. Los modelos matemáticos en muchas ocasiones emplean el cálculo diferencial y o ecuaciones diferenciales las cuales se resuelven dependiendo del tipo de ecuación diferencial, su grado y su orden, además de esto es necesario identificar las condiciones iniciales o de frontera específicos del problema que se resuelve, criterios que le dan a la solución de las ecuaciones diferenciales su característica particular, para determinar las condiciones iniciales en ocasiones se necesita de una gran experiencia para determinarlas de manera apropiada. Por esta razón es de gran importancia Matemática y su impacto en la mayor parte de actividades que realiza el hombre y que contribuye permanentemente a mejorar su calidad de vida y mejorar procesos (22).

Uno de los problemas que resuelve los modelos matemáticos son fenómenos que se repiten con cierta frecuencia los cuales necesitan una respuesta inmediata, los cuales al tener un modelo matemático definido se puede resolver con cierta celeridad disminuyendo tiempos y espacios necesarios en otra actividades.

Los modelos matemáticos además tratan los fenómenos que se resuelven en términos generales, para esto se emplean constantes y variables que deben analizarse en el interior del fenómeno para de esta manera presentar una solución también general, que permita cierta variaciones de dichas constantes. El objetivo principal de la resolución de una ecuación diferencial es la de obtener la función original y esta nos da una idea clara de cuál es el comportamiento del fenómeno, alcanzando mediante la interpretación grafica la comprensión del fenómeno en todos sus instancias, incluso dando la posibilidad de encontrar proyecciones si es del caso.

Según (1), (2) y (3) nos dicen que las ecuaciones diferenciales ordinarias (EDO). Son ecuaciones que relacionan varias funciones también llamadas variables dependientes y sus derivadas, con otras denominadas variables independientes; cuya solución no es otra cosa sino una nueva función que relaciona dichas variables, por lo que es importante diferenciarla de las ecuaciones logarítmicas, aritméticas, exponenciales, entre otras. En este caso de ecuaciones no buscaremos números si no letras. El concepto a primera instancia es muy confuso por lo que tenemos las siguientes palabras clave. Expresión algebraica, ecuación, variable, derivadas y función y ecuaciones diferenciales, las definiciones de estas palabras según (1), (2) y (3) son:

Expresión algebraica. - es un conjunto de constantes y una o más variables que se encuentran relacionadas entre sí a través de operaciones aritméticas y o funciones trigonométricas. 
Ecuación. - Igualdad de dos expresiones algebraicas en las cual se verifica una identidad para cierto valor o valores de las variables en correspondencia con las expresiones algebraicas presentes.

Las ecuaciones dependiendo el tipo de expresión algebraica presente, pueden ser entre otras: polinómicas, trigonométricas, logarítmicas o una combinación de estas.

En las ecuaciones polinómicas, dependiendo del exponente de las variables se puede decir que son de primer grado, segundo grado, etc.

Función. - Es la relación que se verifica entre dos conjuntos, un conjunto denominado de partida y uno de llegada, esta relación se verifica de tal manera que para cada elemento del conjunto de partida le corresponde uno y solamente uno del conjunto de llegada, la relación que establece el nexo entre los elementos de los conjuntos se establece a través de una expresión algebraica a la cual se denomina función. Dependiendo de la expresión algebraica la función puede ser exponencial, logarítmica, trigonométrica, o una combinación entre ellas. La relación entre las variables se determina de tal manera que existe una de ellas llamada independiente (que puede tomar el valor independientemente) y la otra variable dependiente (su valor depende de la variable independiente y su valor se calcula a partir de la relación presente). En ocasiones se emplea la nomenclatura $\mathrm{f}(\mathrm{x})$, para referirse a una función de la variable independiente.

Derivada. - Es la tasa de crecimiento de una de las variables (dependiente) respecto de la otra variable (independiente), es decir el valor límite entre el aumento del valor de una función y el aumento de $x$.

Ecuación diferencial. - Es una ecuación donde existe o están presentes una o más derivadas de una función. Se dice que una función diferencial es ordinaria, si la función presente depende de una variable, es decir tiene una sola variable independiente, si la función depende de más de una variable entonces la ecuación se llama ecuación diferencial parcial. Matemáticamente encontrar la solución de la ecuación diferencial es encontrar la función original o primitiva, la cual se encuentra derivada en la ecuación diferencial.

Orden de una ecuación diferencial. El orden de una ecuación diferencial está dado por el mayor orden de la derivada que se encuentra presenta en la ecuación diferencial.

Las ecuaciones diferenciales, maneja también el termino de diferencial relacionado con las variables (dx, dy, dt, dv, etc.), las cuales se debe entender que son la variación o incremento de dicha variable cuando esta variación tiende a ser cero, en otras palabras, es una variación sumamente pequeña.

\section{Solución de la ecuación diferencial.}


La solución de las ecuaciones diferenciales implica el conocimiento de las diferentes técnicas de solución dependiendo del tipo de ecuación diferencial, y de su forma, todas estas soluciones pueden ser consideradas como soluciones generales, en donde las constantes de integración indefinida están presentes para luego ser calculadas a partir de la condiciones iniciales o frontera, las cuales deben ser información recopilada del fenómeno físico analizado.

Para hablar de modelos matemáticos debemos comprender algunos términos que son muy utilizados como: modelo, sistema, sistema dinámico, entre otros.

Una vez identificado el problema, se abordan los métodos de solución existentes.

\section{Suavizado Estadístico}

Los Métodos no Paramétricos son herramientas estadísticas de inferencia que se encargan de estudiar los modelos estadísticos cuya distribución de probabilidad (F) no es conocida y por lo tanto no dependen de ella (Distribution-Free). (23)

\section{Estimación no Paramétrica de Curvas.}

Según (Delicado 2008), la estimación no paramétrica de curvas son técnicas que permiten estimar funciones relacionadas con la distribución de probabilidad de los datos. Por ejemplo se puede tener interés en estimar la función de distribución $\mathrm{F}(x)$, la función de densidad $f(x)$, la tasa de fallo,$\lambda(x)=f(x) / 1-\mathrm{F}(x)$, la función de regresión $m(x)=E(Y \mid X=x)$ o la varianza condicional $\sigma^{2}(x)=V(Y \mid X=x)$.

\section{Exploración de las densidades.}

En ésta sección nos dedicaremos a estimar las densidades de cada período de la variable Speed, para esto es conveniente mencionar la función "sm.density" que se encuentra en la librería "sm".

\section{Función sm.density}

sm.density(x, h, h.weights, model, display, panel, positive, ...)

Permite la estimación de la densidad no paramétrica en una o dos dimensiones. El único argumento requerido es el dato $\mathrm{x}$, que puede ser una lista en el caso unidimensional, o una lista de listas o una matriz con dos columnas en el caso bidimensional. El argumento de palabra clave: h proporciona el parámetro de suavizado (o dos parámetros de suavizado en el caso bidimensional, uno para cada dimensión), y si se omite este parámetro se usa un parámetro de suavizado óptimo normal (en el caso bidimensional el parámetro de suavizado predeterminado) se calcula por separado para cada dimensión). (24) 
El histograma nos muestra la forma de la distribución de un conjunto de datos y su utilidad radica en el hecho de que indica la forma de la función de densidad subyacente. Del lado derecho tendremos en cambio una forma alternativa de la función de densidad expresada como una curva suave. Es notable mencionar que hacemos la comparación de histograma vs curva suavizada para enfatizar en la gran ventaja que implica describir curvas suavizadas ya que brinda una mejor comprensión de la distribución y permite comparar dichas densidades.

\section{Comparación de las estimaciones de densidades.}

Haremos comparaciones de a pares para una mejor comprensión y lo haremos a través de otra función que se puede encontrar en el paquete "sm", hablamos de la función "sm.density.compare".

\section{Función sm.density.compare}

sm.density.compare(x, group, model...)

Esta función permite comparar dos o más estimaciones de densidades univariadas. El valor predeterminado del parámetro del modelo es "none", en cuyo caso las densidades simplemente se dibujan en un conjunto común de ejes. Si el modelo se establece en "equal", se lleva a cabo una prueba de arranque de igualdad. Si la comparación implica sólo dos funciones de densidad, la prueba irá acompañada de una banda de referencia gráfica para la igualdad. (25)

Se cuenta entonces con un medio para identificar si las diferencias entre las estimaciones reflejan diferencias sistemáticas en las distribuciones subyacentes, o si podrían atribuirse simplemente a la variación aleatoria.(26)

Formalmente las hipótesis que se plantean así:

$$
\begin{aligned}
& H_{o}: f(y)=g(y), \quad \text { paratodas las } y . \\
& H_{1}: f(y) \neq g(y), \quad \text { paraalguna } y .
\end{aligned}
$$

En la Sección 2.5 del libro de Bowman and Azzalini, 1997, se propuso una estadística integrada de error al cuadrado para comparar una estimación de densidad con una curva normal. Un enfoque análogo para comparar dos estimaciones de densidad $\hat{f} y \hat{g}$ es a través de la estadística:

$$
\int\{\hat{f}(y)-\hat{g}(y)\}^{2} d y
$$


En particular las medias de las estimaciones son:

$$
\begin{aligned}
& \mathbb{E}\{\hat{f}(y)\}=\int \phi(y-z ; h) f(z) d z \\
& \mathbb{E}\{\hat{g}(y)\}=\int \phi(y-z ; h) g(z) d z
\end{aligned}
$$

Bajo la hipótesis nula de que las dos funciones de densidad f y g son idénticas, estas dos medias también serán idénticas si se usa el mismo parámetro de suavizado " $h$ " en la construcción de cada uno. El contraste de las estimaciones $\hat{f}-\hat{g}$ tendrá una media de 0 bajo la hipótesis nula.

Bajo hipótesis nula, la asignación de etiquetas de grupo es completamente aleatoria, ya que ambos grupos de datos se generan a partir de la misma función de densidad subyacente. La distribución de la estadística $\int\{\hat{f}(y)-\hat{g}(y)\}^{2} d y$ bajo la hipótesis nula se puede construir fácilmente mediante la evaluación de la estadística sobre los datos producidos por la permutación aleatoria de las etiquetas del grupo.

En el contexto actual, una banda de referencia debería permitir comparar dos estimaciones de densidad $\hat{f}$ y $\hat{g}$. El nivel de significancia es $\alpha=0.05$. Un medio simple de mostrar la separación gráficamente es superponer en una gráfica de las estimaciones una banda de referencia que está centrada en el promedio de las dos curvas y cuyo ancho en el punto "y" es dos errores estándar.(26).

\section{Ecuación de balance}

Los fluidos, por definición, pueden fluir, pero son esencialmente incompresibles. Esto proporciona información muy útil sobre cómo se comportan estos cuando fluyen a través de una tubería o un río. Considérese una manguera cuyo diámetro disminuye a lo largo de su longitud, la "ecuación de continuidad" es una consecuencia directa del hecho más bien trivial de que lo que entra en la manguera debe salir (27). El volumen de agua que fluye a través de la manguera por unidad de tiempo. La tarea principal en dinámica de fluidos es encontrar el campo de velocidad que describa el flujo en un dominio dado, para hacer esto, uno se usa las ecuaciones básicas del flujo de fluidos.

A escala microscópica, el fluido comprende moléculas individuales y sus propiedades físicas. (densidad, velocidad, etc.) son violentamente no uniformes. Sin embargo, los fenómenos estudiados en dinámica de fluidos son macroscópicos, por lo que generalmente no tomamos este detalle molecular en cuenta (28). En lugar de eso, se trata el fluido como un continuo al 
verlo lo suficientemente mas denso que cualquier elemento fluido "pequeño", en realidad este todavía contiene muchas moléculas. Uno luego puede asignar una velocidad de flujo masivo local $\mathrm{v}(\mathrm{x}, \mathrm{t})$ al elemento en el punto $\mathrm{x}$, promediando sobre las velocidades moleculares brownianas, mucho más rápidas y violentamente fluctuantes. Del mismo modo, se define una densidad promediada localmente $\rho(\mathrm{x}, \mathrm{t})$, etc. Estas cantidades promediadas localmente entonces varían suavemente con x en la escala macroscópica del flujo(26).

Metodología

Unidad de análisis

Datos del monitoreo de $\mathrm{SO} 2$

Factor

Reducción matemática

Variable respuesta

Modelo físico-matemático de flujo de SO2

Tipo de investigación

El estudio fue aplicado a las ciencias básicas y atmosféricas.

Diseño

Experimental

Nivel de la investigación

Exploratorio, descriptivo, explicativo y predictivo.

Metodología de la investigación

El método científico fue la base del presente en todo el desarrollo del trabajo experimental.

Métodos específicos de investigación: Pararosanilina: absorción en medio líquido y análisis colorimétrico posterior.

Analizador continúo por fluorescencia.

Método pasivo referido en la norma europea EN 13528-1: 2002. EN 13528-2:2002 EN 13528-3:2002

Enfoque de la investigación 
La investigación tiene un enfoque mixto; cualitativo y cuantitativo.

Temporalidad de la investigación

El estudio tiene como como característica la temporalidad longitudinal, pues se compilaron datos a través de diferentes momentos de la investigación.

\section{Población}

La población y muestra correspondió a 96 datos de monitoreo.

Técnicas e instrumentos

Técnica: Observación estructurada; instrumento: inventario.

Tratamiento de datos

Se aplicaron métodos matemáticos (análisis vectorial y ecuaciones diferenciales), físicos (ecuación de balance y continuidad) y suavizado estadístico mediante el programa Infostat.

\section{Resultados}

\section{Modelo de flujo}

Análisis de densidad y concentración del $\boldsymbol{S O}_{2}$

La prevalencia de SO2 en el punto de monitoreo equivale al modelo (29)

$C=\int_{R} \rho d v$

C: Concentración de $\mathrm{SO}_{2}$.

$\boldsymbol{\rho}$ : Densidad del $\mathrm{SO}_{2}$.

$d v=$ Diferencial de velocidad

Si se hace variar la concentración de $\mathrm{SO}_{2}$ con respecto al tiempo, la ecuación diferencial queda en términos del tiempo, entendiéndose que la concentración de $\mathrm{SO}_{2}$ en función del tiempo está dada por la ecuación diferencial dada:

$\frac{d C}{d t}=\int_{R}-\frac{\partial \rho}{\partial t} d v$

La producción de SO2 debido a fuentes móviles "G":

$\boldsymbol{G}=\int_{R} g d v$ 
G: Producción de $\mathrm{SO}_{2}$ fuentes móviles.

El flujo de SO2 por unidad de superficie se denota por “ $\mathbf{j}$ ”; $\mathbf{n}$ corresponde al vector normal, el cual multiplicado en producto punto por " $\mathrm{j}$ ", garantiza un flujo perpendicular a la superficie por donde atraviesa el contaminante (30).

$\int_{S} \vec{J} \cdot \vec{n} d s=\int_{R} \vec{\nabla} \cdot \vec{J} d v$

$\vec{J}=\rho \cdot \vec{v}$

$\vec{v}$ : Es la velocidad.

Ecuación de balance

$\int_{R}\left(-\frac{\partial \rho}{\partial t}-G-\vec{\nabla} \cdot \vec{J}\right) d v=0$

De lo anterior se desprende que:

$\vec{\nabla} \cdot \vec{J}=-\frac{\partial \rho}{\partial t}-G$

La ecuación de flujo del $\mathrm{SO}_{2}$ se relaciona con la razón de cambio (31) de la densidad con respecto al tiempo y a la producción de $\mathrm{SO}_{2}$.

En el caso de inversión térmica.

$\vec{\nabla} \cdot \rho \vec{v}=0$

$\vec{\nabla} \cdot \vec{v}=0$

La velocidad es solenoidal; el contaminante queda prevalente en la atmósfera "girando" sobre su propio eje.

Asociando los valores del $\mathrm{SO}_{2}$ con respecto a su concentración, temperatura ambiente a distintas horas y considerando la presión atmosférica en la ciudad de Riobamba, se determina la densidad del $\mathrm{SO}_{2}$ a cada hora mediante el siguiente modelo:

$\rho=\frac{P . M}{R \cdot T}$

De donde:

P: Es la Presión Atmosférica en la ciudad de Riobamba.

M: Es la masa molecular del dióxido de Azufre.

R: Es la constante de los gases ideales. 


\section{T: Es la temperatura ambiente a la hora indicada.}

A continuación, se muestra en la Tabla 2 los valores de las densidades calculadas a las distintas horas.

Realizando el gráfico de Dispersión de la Densidad vs Tiempo se obtiene el siguiente gráfico:

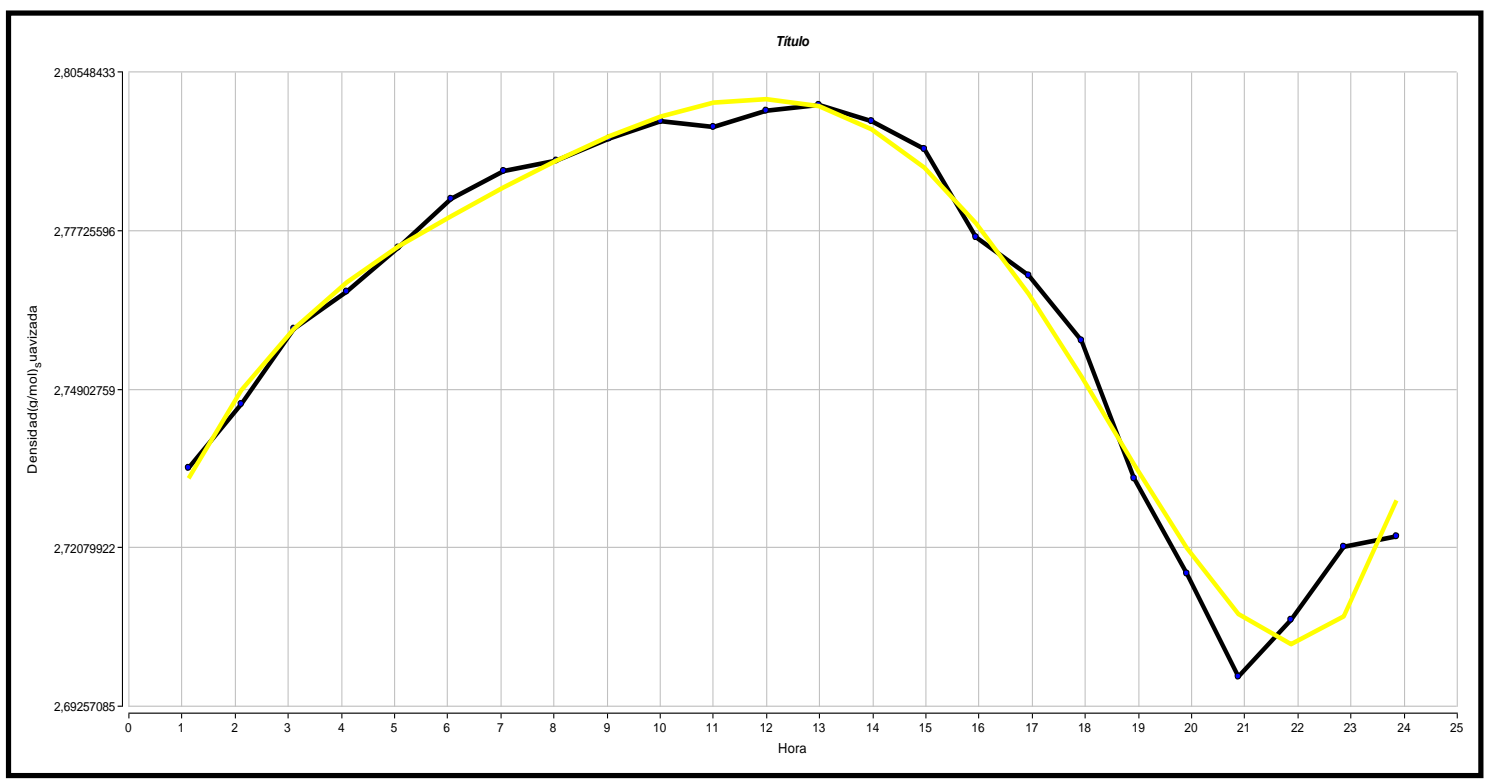

Fig. 2: Densidad del SO2 con respecto al tiempo, se toma la hora 1 como las 17:00 y técnica de suavizado polinomial de grado 5 .

Se aprecia en la gráfica dos líneas, la primera de color negro es la gráfica empírica de la Densidad vs Tiempo, por otro lado, la gráfica de color amarillo es una aproximación mediante suavizado (32) por técnica polinomial de grado 5. El polinomio en cuestión es el siguiente:

$\rho=6.3 \times 10^{-7} t^{5}-3.4 \times 10^{-5} t^{4}+0.0006 t^{3}-0.0057 t^{2}+0.0294 t+2.7084$

El polinomio es suave (33) por lo tanto derivable, la función inyectiva (Causa-Efecto), continua a lo largo de la línea de tiempo y presenta un máximo a las 6:00 con $2.799478179368 \mathrm{~g} / \mathrm{mol}$.

Es de interés también determinar un modelo matemático aproximado de la concentración de $\mathrm{SO} 2$ (ug/m3) con respecto a la hora del día (tiempo), se adjunta la gráfica siguiente. 


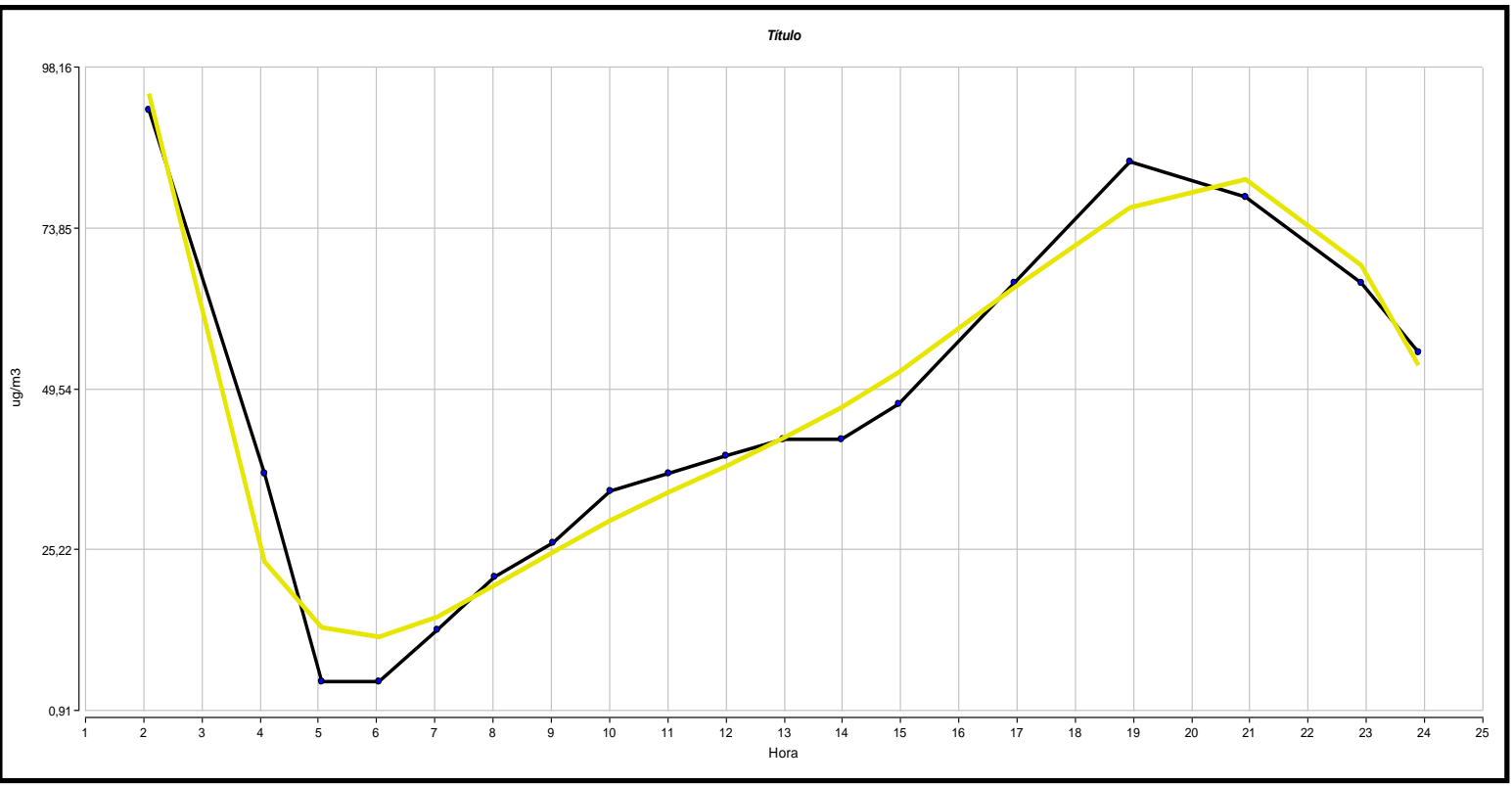

Gráfica 2: Concentración del SO2 con respecto al tiempo, se toma la hora 1 como las 17:00 y técnica de suavizado polinomial de grado 5.

Se aprecia en la gráfica dos líneas, la primera de color negro es la gráfica empírica de la Concentración del SO2 vs Tiempo, por otro lado, la gráfica de color amarillo es una aproximación mediante suavizado por técnica polinomial de grado 5. El polinomio en cuestión es el siguiente:

$$
G=-0.0009 t^{5}+0.0612 t^{4}-1.6042 t^{3}+20.158 t^{2}-116.04 t+256
$$

\section{MODELO DE FLUJO DE $\mathrm{SO}_{2}$}

$\vec{\nabla} \cdot \vec{J}=-\frac{\partial\left(6.3 \times 10^{-7} t^{5}-3.4 \times 10^{-5} x^{4}+0.0006 t^{3}-0.0057 t^{2}+0.0294 t+2.7084\right)}{\partial t}-0.0009 t^{5}+$

$0.0612 t^{4}-1.6042 t^{3}+20.158 t^{2}-116.04 t+256$

\section{Conclusiones.}

- Se desarrolló el modelo de flujo de SO2 en un punto crítico del Centro Histórico de Riobamba como lo es el Parque Maldonado debido a la notable concurrencia de fuentes móviles; dicho modelo dependiente de la densidad y el tiempo predice el desplazamiento del contaminante en el tiempo, dicho flujo.

- La función de densidad de SO2 muestra una variabilidad con respecto al tiempo; el polinomio es suave, por lo tanto derivable, la función es inyectiva, demostrando causa-efecto, continua a lo largo de la línea de tiempo y presenta un mínimo a las 21:00 y un máximo a las 13:00.

- La función de concentración del $\mathrm{SO} 2$, corresponde a la función polinómica de $5^{\circ}$ grado:

$G=-0.0009 t^{5}+0.0612 t^{4}-1.6042 t^{3}+20.158 t^{2}-116.04 t+256$, suave sin 
ajuste, inyectiva, registrando un máximo a las 2:00 y un mínimo de 5 a 6 (excepto en el suavizado)

\section{Referencias bibliográficas.}

Zill, Dennis. Ecuaciones diferenciales . Mexico : Thomson, 2010.

Aguirregabiria, Juan. Ecuaciones diferenciales ordinarias para estudiantes de Física . España : Universidad de pais de vasco, 2000.

Ecuaciones diferenciales. Lemoine, Carlos. 1965, Revista colombiana de matematicas, págs. 10-11.

LOUIS, Jean-François. A parametric model of vertical eddy fluxes in the atmosphere. Boundary-Layer Meteorology. 1 September 1979. Vol. 17, no. 2, p. 187-202. DOI 10.1007/BF00117978.

$\mathrm{HE}$, Jie. Pollution haven hypothesis and environmental impacts of foreign direct investment: The case of industrial emission of sulfur dioxide (SO2) in Chinese provinces. Ecological Economics. 1 November 2006. Vol. 60, no. 1, p. 228-245. DOI 10.1016/j.ecolecon.2005.12.008.

PANDEY, Jai Shanker, KUMAR, Rakesh and DEVOTTA, Sukumar. Health risks of NO2, SPM and SO2 in Delhi (India). Atmospheric Environment. 1 November 2005. Vol. 39, no. 36, p. 68686874. DOI 10.1016/j.atmosenv.2005.08.004.

PEARL, J., HANEL, R., KUNDE, V., MAGUIRE, W., FOX, K., GUPTA, S., PONNAMPERUMA, C. and RAULIN, F. Identification of gaseous SO2 and new upper limits for other gases on lo. Nature. 1 August 1979. Vol. 280, p. 755-758. DOI 10.1038/280755a0.

RIDDER, Koen de, VIAENE, Peter, VEL, Karen van de, BRASSEUR, Olivier, CHEYMOL, Anne and FIERENS, Frans. The impact of model resolution on simulated ambient air quality and associated human exposure. Atmósfera. October 2014. Vol. 27, no. 4, p. 403-410. DOI 10.1016/S0187-6236(14)70038-4.

Silvio álvarez luna. Modelamiento de la dispersión del dióxido de azufre, y su relación con la calidad del aire del centro histórico de la ciudad de Riobamba - Ecuador. UNSM, 2017.

WARE, J. H., FERRIS, Jr BG, DOCKERY, D. W., SPENGLER, J. D., STRAM, D. O. and SPEIZER, F. E. Effects of ambient sulfur oxides and suspended particles on respiratory health of preadolescent children. The American review of respiratory disease. May 1986. Vol. 133, no. 5, p. 834-842.

Comparación del flujo de emisión de SO2 derivadas de COSPEC y MODIS y su complementariedad en el monitoreo volcánico: Caso de estudio en el Volcán Popocatépetl (México). [online]. [Accessed 20 January 2019]. 
BOICHU, Marie, CLARISSE, Lieven, PÉRÉ, Jean-Christophe, HERBIN, Hervé, GOLOUB, Philippe, THIEULEUX, François, KHVOROSTYANOV, Dmitry, DUCOS, Fabrice, WINIAREK, Victor, CLERBAUX, Cathy and TANRÉ, Didier. Reconstruction of flux and altitude of volcanic SO2 emissions from IASI satellite observations: implications for volcanological and atmospherical studies. In : 4th IASI International Conference [online]. 11 April 2016. [Accessed 20 January 2019].

PATIA, H., EGGINS, S. M., ARCULUS, R. J., MCKEE, C. O., JOHNSON, R. W. and BRADNEY, A. The 1994-2001 eruptive period at Rabaul, Papua New Guinea: Petrological and geochemical evidence for basalt injections into a shallow dacite magma reservoir, and significant $\mathrm{SO} 2$ flux. Journal of Volcanology and Geothermal Research. 1 October 2017. Vol. 345, p. 200-217. DOI 10.1016/j.jvolgeores.2017.08.011.

Ponce, Patricia. Estimación de las emisiones de dióxido de azufre - so2, asociadas con el crecimiento de un domo de lava en el volcán galeras EN 2008. Boletín de Geología. 24 February 2017. Vol. 39, no. 1, p. 69-81.

JIMÉNEZ. Comparación del flujo de emisión de SO2 y su complementariedad en el monitoreo volcánico. ResearchGate [online]. 2018. [Accessed 20 January 2019]. Available from: https://www.researchgate.net/publication/327762805_Comparacion_del_flujo_de_emision_de_S O2_derivadas_de_COSPEC_y_MODIS_y_su_complementariedad_en_el_monitoreo_volcanico_Cas o_de_estudio_en_el_Volcan_Popocatepetl_MexicoPDF |

PÉREZ. Erupción del 3 de marzo de 2015 en el volcán Villarrica caracterización del lahar del estero correntoso y cuantificación de emisiones post-eruptivas de SO2. [online]. 2018.

[Accessed 20 January 2019]. Available from: http://repositorio.udec.cl/handle/11594/3098

Oxidation of $\mathrm{SO} 2$ in rainwater and its role in acid rain chemistry - ScienceDirect. [online]. [Accessed 20 January 2019].

SULFUR DIOXIDE -- Industrial Uses. [online]. 2018. [Accessed 20 January 2019]. Available from:http://scorecard.goodguide.com/chemicalprofiles/uses.tcl?edf_substance_id=7446\%2D09\%2D5

SO2 scrubbing technologies: A review - Srivastava - 2001 - Environmental Progress - Wiley Online Library. [online]. [Accessed 20 January 2019]. Available from: https://onlinelibrary.wiley.com/doi/abs/10.1002/ep.670200410

Chicago Air Pollution Study: SO2 Levels and Acute Illness in Patients With Chronic Bronchopulmonary Disease: Archives of Environmental Health: An International Journal: Vol 18, No 5. [online]. 2013.

A review of controlled human SO2 exposure studies contributing to the US EPA integrated science assessment for sulfur oxides: Inhalation Toxicology: Vol 23, No 1. [online]. [Accessed 20 January 2019]. 
Ecuaciones diferenciales ordinarias - José Carlos Bellido Guerrero, Alberto Donoso Bellón, Sebastián Lajara López - Google Libros. [online].

Ecuaciones diferenciales en derivadas parciales: con métodos de variable ... - Hans F. Weinberger - Google Libros. [online]. [Accessed 24 January 2019].

Diseño de circuitos y sistemas integrados - José Antonio Rubio Sola - Google Libros. [online]. [Accessed 25 January 2019].

Fisica/ Physics - Miguel Nunez - Google Libros. [online]. [Accessed 25 January 2019]. Available from:

Suavizado estadístico [online]. [Accessed 25 January 2019]. Available from: http://dspace.ucbscz.edu.bo/dspace/bitstream/123456789/13117/1/9970.pdf

Métodos de suavizado estadístico [online]. [Accessed 25 January 2019]. Available from: http://e-spacio.uned.es/fez/eserv/bibliuned:editorial-Educacionpermanente0186080EP02A01/Documento_0186080EP02A01.pdf

MARTínEZ-CAMBLOR, Pablo. Estudio sobre los efectos del parámetro de suavizado en contrastes no paramétricos para k-muestras. Revista Colombiana de Estadística. 2008. P. 12.

Applied Smoothing Techniques for Data Analysis: The Kernel Approach with S ... - Adrian W. Bowman, Adelchi Azzalini - Google Libros. [online]. [Accessed 25 January 2019].

Ecuación de continuidad.pdf [online]. [Accessed 24 January 2019]. Available from: https://community.dur.ac.uk/suzanne.fielding/teaching/BLT/sec1.pdf

Continuity Equation. [online]. 2018. [Accessed 24 January 2019]. Available from: http://www.fsl.orst.edu/geowater/FX3/help/8_Hydraulic_Reference/Continuity_Equation.htm

HWEI. P. HSU. Análisis vectorial para ciencias e ingeniería. Simon \& Shuster, 1969.

Vector analysis | mathematics | Britannica.com. [online]. [Accessed 20 January 2019]. Available from: https://www.britannica.com/science/vector-analysis

CASTILLO, Ing Mario. Vectorial Analysis. Palibrio, 2012. ISBN 978-1-4633-2977-8. The solution for the problems presented in this book are solved with algebra, analytic geometry, differential and integral calculus geometry, MATLAB and vector analysis.Google-Books-ID: FeDopqjcQEsC

OLAYA. Modelamiento estadístico. Univ. Nacional de Colombia, 2005. ISBN 978-958-701559-1. Google-Books-ID: 1UGDhYbvzYAC

JOHNSONBAUGH, Richard. Matemáticas discretas. Pearson Educación, 2005. ISBN 978970-26-0637-6. 


\section{Para citar el artículo indexado.}

Mendoza J., Baquero J., Ricardo E., Insuasti R. (2019) Aplicación de la mecánica de fluidos en la modelación matemática de flujo de SO2 en el Centro Histórico de Riobamba Ecuador. Revista electrónica Ciencia Digital 3(2), 289-305. Recuperado desde: http://cienciadigital.org/revistacienciadigital2/index.php/CienciaDigital/article/view/467/1064

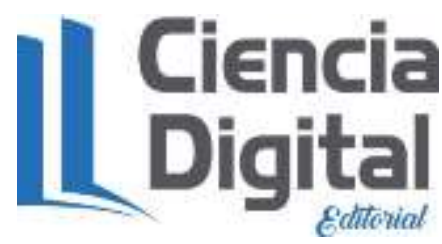

El artículo que se publica es de exclusiva responsabilidad de los autores y no necesariamente reflejan el pensamiento de la Revista Ciencia Digital.

El articulo queda en propiedad de la revista y, por tanto, su publicación parcial y/o total en otro medio tiene que ser autorizado por el director de la Revista Ciencia Digital.
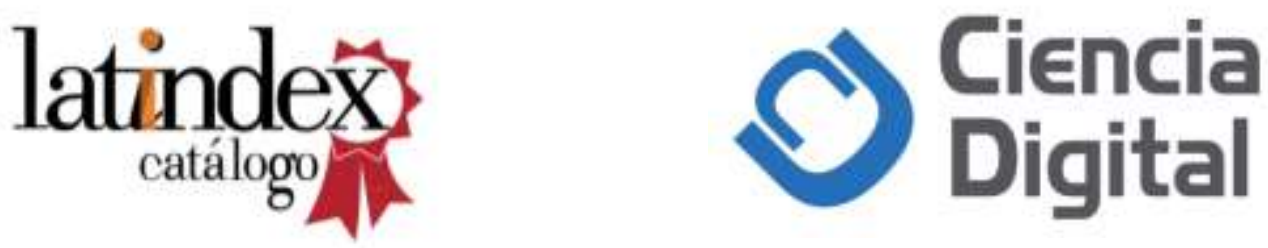